\title{
Desorption Electrospray Ionization (DESI) Analysis of Tryptic Digests/Peptides
}

\author{
Zoltan Takats, Justin M. Wiseman, Demian R. Ifa, and R. Graham Cooks
}

This protocol was adapted from "Desorption Electrospray lonization: Proteomics Studies by a Method that Bridges ESI and MALDI," Chapter 6, in Proteomics: Methods Express (eds. O'Connor and Hames). Scion Publishing Ltd., Oxfordshire, UK, 2007.

\section{INTRODUCTION}

The analytical utility of desorption electrospray ionization (DESI) is such that it can be applied to qualitative proteomics research in the same way as matrix-assisted laser desorption/ionization (MALDI) and electrospray ionization (ESI) methods, although little work has yet been reported in this regard. Because DESI is a surface analysis technique and easily automated, it can be implemented for highthroughput applications, which include the analysis of chromatographic fractions of digested proteins. The analysis of tryptic peptides follows the same protocols as in typical MALDI or ESI methods, except that the mixture is spotted directly onto an insulating surface, allowed to dry, and analyzed directly without adding matrix compounds (as in the case of MALDI methods). The spectral characteristics are similar to those of ESI in that both singly and multiply charged analyte ions are detected. Spectra are highly similar to electrospray spectra of tryptic digests with regard to the overwhelming presence of multiply charged ions of peptides. DESI-mass spectrometry (DESI-MS) is an emerging technique with great promise, but its application range is still being investigated. Therefore, the protocol for DESI-MS analysis of tryptic digests/peptides presented here provides general procedures used for the applications that have been investigated so far. Optimal ion source parameters and surface types may vary, depending on the application.

\section{RELATED INFORMATION}

An introduction to DESI instrumentation, methods, and applications is provided in the CSH Protocols article Desorption Electrospray lonization: Proteomics Studies by a Method that Bridges ESI and MALDI (this issue). Protocols for Desorption Electrospray Ionization (DESI) Analysis of Intact Proteins/Oligopeptides and In Situ Desorption Electrospray lonization (DESI) Analysis of Tissue Sections are also available (this issue).

Recently, Creaser and coworkers (Kaur-Atwal et al. 2007) demonstrated the utility of the combination of DESI and ion mobility spectrometry/MS for the rapid analysis of tryptic peptides without sample pretreatment or chromatographic separation. The results of their studies showed good agreement between DESI and ESI analysis of a tryptic digest of bovine serum albumin in terms of absolute signal intensity and signal-to-noise $(\mathrm{S} / \mathrm{N})$ ratio. Interestingly, their results also showed that, in DESI, there were more singly charged species detected than in the case of ESI, resulting in a less complex spectrum in the mass range between mass-to-charge ratio $(\mathrm{m} / \mathrm{z}) 650$ and 800 . Consequently, this resulted in better sequence coverage with higher confidence in peptide matches for protein identification. The analysis of a trypsin digest of cytochrome $c$ is shown in Figure $1 \mathrm{~A}$, and the product ion MS/MS spectrum of one tryptic peptide is shown in Figure 1B. This is one example of the type and quality of mass spectrum recorded using DESI-MS without chromatographic separation. 


\section{MATERIALS}

CAUTIONS AND RECIPES: Please see Appendices for appropriate handling of materials marked with $<!>$, and recipes for reagents marked with $<\mathbf{R}>$.

\section{Reagents}

Spray solvent, such as one of the following:

$<$ ! $>$ Aqueous buffers (e.g., $10 \mathrm{mM}$ ammonium acetate $\left[\mathrm{H}_{3} \mathrm{CCOONH}_{4}\right]$ )

$<!>\mathrm{H}_{2} \mathrm{O}$ /acetonitrile mixtures at various compositions

$<!>\mathrm{H}_{2} \mathrm{O} /$ methanol mixtures at various compositions

Do not use nonvolatile buffers or high concentrations of acid (e.g., acetic acid).

Tryptic digest/peptides (1-100 ng/mL)

$<$ ! > Aqueous buffers or buffered $\mathrm{H}_{2} \mathrm{O} /$ acetonitrile mixtures can be used for deposition (Step 1). Methanol or methanol-containing solvent systems are not compatible with the polymethyl methacrylate (PMMA) surface.

\section{Equipment}

Mass spectrometer equipped with a DESI ion source

Pipette tips

Surface slides (PMMA or polytetrafluoroethylene [PTFE])

The use of other surface materials is not advised.

\section{METHOD}

1. Deposit a 1- $\mu \mathrm{L}$ aliquot of a solution of tryptic digest onto a PMMA or PTFE slide.

The ideal surface concentration is $10 \mathrm{ng} / \mathrm{mm}^{2}$; higher concentrations cause disadvantageous suppression effects.

2. Allow the peptide sample spot to dry.

3. Expose the dried sample spot to a pneumatically assisted electrospray using a DESI ion source coupled to a mass spectrometer; typical parameters used for analysis of tryptic digests/peptides are as follows:

\begin{tabular}{ll} 
Parameter & Value \\
\hline Spray tip-to-surface distance & $2-3 \mathrm{~mm}$ \\
Incident angle of spray & $60^{\circ}-80^{\circ}$ \\
Spray tip-to-MS inlet distance & $3-5 \mathrm{~mm}$ \\
Collection angle & $0^{\circ}-5^{\circ}$ \\
Solvent flow rate & $1-2 \mu \mathrm{L} / \mathrm{min}$ \\
Nebulizing gas linear velocity & $300-400 \mathrm{~m} / \mathrm{sec}$ \\
Spray high voltage & $4-6 \mathrm{kV}$ \\
Surface temperature & $50^{\circ} \mathrm{C}-100^{\circ} \mathrm{C}$
\end{tabular}

DESI spectra show ion abundances that vary with time, similar to other desorption ionization (DI) methods. The signal can be stabilized by using higher spray tip-to-surface distances at lower incident angles. In optimal cases, spectra can be collected for 20-100 sec without moving the surface in the case of deposited samples.

See Troubleshooting.

\section{TROUBLESHOOTING}

Problem: There are no surface originated ions in the spectra.

[Step 3]

Solution: A possible cause of this is that the sprayed droplets and ions are not reaching the surface because of incorrect spray parameters (i.e., the volumetric flow rate is too low or there is no applied high voltage) or surface charging effects. Always check the spray pattern on the surface. If the spray 

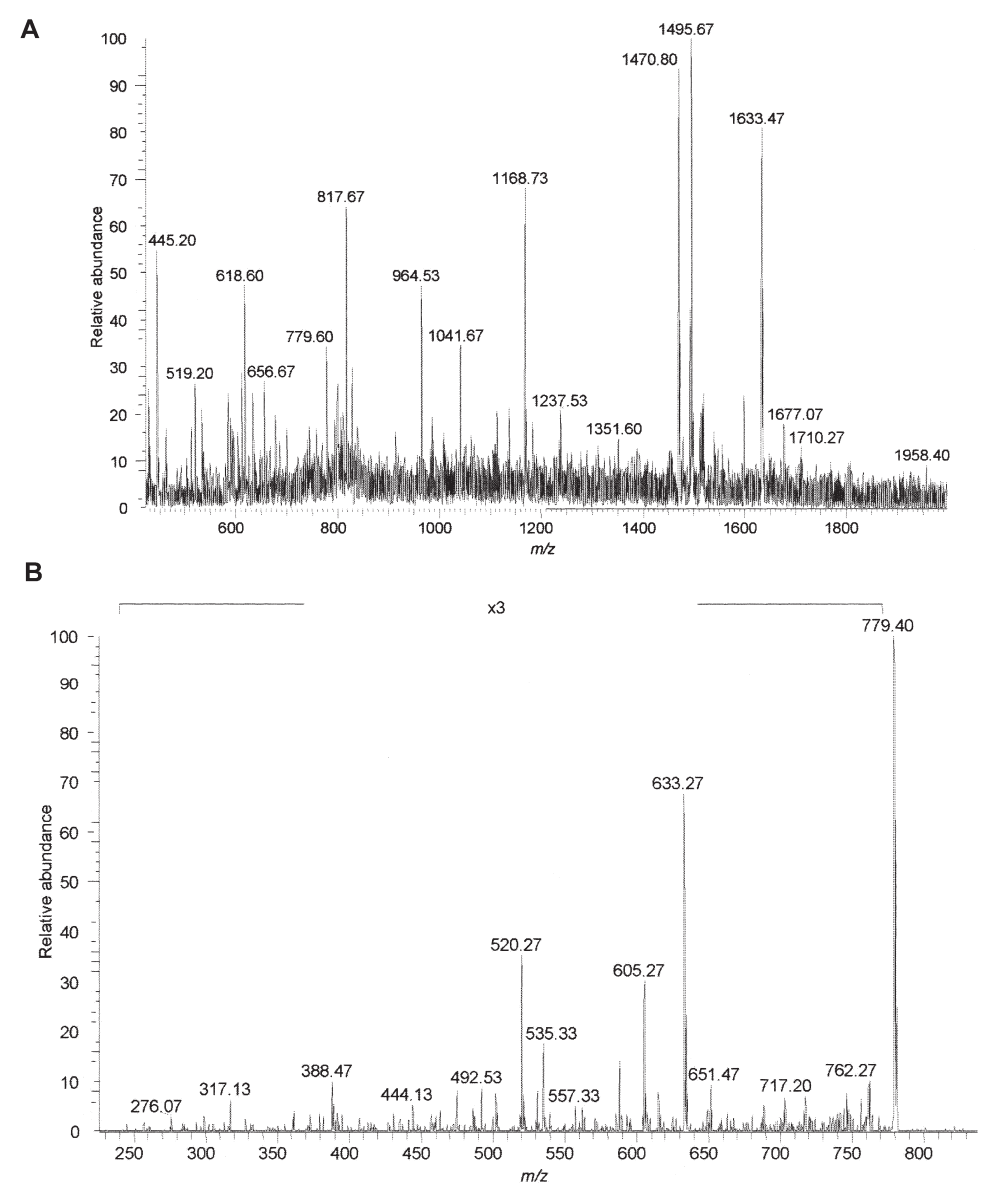

FIGURE 1. DESI mass spectra of a trypsin digest of cytochrome c. (A) DESI mass spectrum of a trypsin digest of a 100ng solution of cytochrome $c$ deposited onto a PMMA surface. Methanol: $\mathrm{H}_{2} \mathrm{O}(1: 1)$ was used as the spray solvent at a flow rate of $5 \mu \mathrm{L} / \mathrm{min}$. (B) MS/MS spectrum of a tryptic fragment at $\mathrm{m} / \mathrm{z} 779$. (Reprinted with permission, (C) 2007 Scion Publishing Ltd.)

is not visible on a glass surface, check to ensure that the solvent syringe pump is on and that there are no blockages in the solvent delivery line that would restrict flow.

Problem: No signal is detected.

[Step 3]

Solution: There may be no spray present, or the sprayed species are being deflected by the charged surface.

Try the following:

1. Increase solvent and gas flow rates.

2. If there is no change, remove the surface and check whether the spray produces ions. Test the spray with $10 \mathrm{mg} / \mathrm{mL}$ bovine cytochrome $c$ in $10 \mathrm{mM}$ aqueous ammonium acetate. The test solution should give a narrow charge state distribution of the protein, with a main charge state of +7 or +8 . If there are no ions, check whether the spray tip (or solvent line) is clogged. If the cytochrome $c$ spectra are full of adducts, change the spray tip.

Problem: There is excessive adduct formation.

\section{[Step 3]}

Solution: This may be caused by insufficient desolvation due to the presence of large droplets or contamination of the surface. Try the following:

1. Increase the gas flow rate and/or heat the surface. 
2. Check whether the sample contains salts at high concentration. If the deposited sample contains more than $100 \mathrm{mM}$ inorganic, nonvolatile salt, dilute it or remove the salts prior to deposition.

3. If a PMMA surface is used in combination with methanol (as solvent for the deposited sample), let the surface dry for an additional $10 \mathrm{~min}$ at $50^{\circ} \mathrm{C}$.

Problem: A transient signal, not suitable for MS/MS, is observed.

[Step 3]

Solution: A possible cause of this is low surface concentration or lack of sample adhesion to the surface. The following are possible solutions:

1. Try a different surface material or a roughened surface.

2. Increase the surface concentration of the sample.

3. Increase the spray tip-to-surface distance.

4. Decrease the solvent flow rate.

Problem: Results exhibit strong suppression effects and poor spectral resolution.

[Step 3]

Solution: Possible causes are high surface concentration or an inappropriate solvent system. Try the following:

1. Decrease the surface concentration of the sample.

2. Change the solvent composition.

\section{REFERENCES}

Kaur-Atwal, G., Weston, D.I., Green, P.S., Croland, S., Bonner, P.L.R. and Creaser, C.S. 2007. Analysis of tryptic peptides using desorption electrospray ionisation combined with ion mobility spec- trometry/mass spectrometry. Rapid Commun. Mass Spectrom. 21: 1131-1138. 


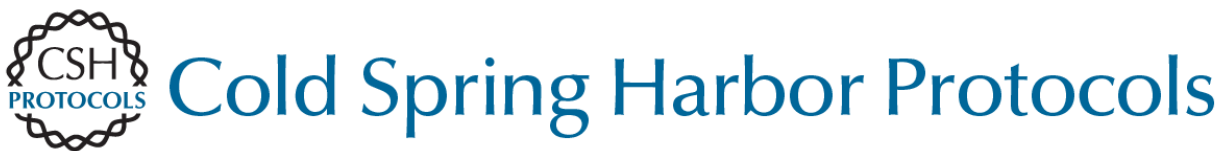

\section{Desorption Electrospray lonization (DESI) Analysis of Tryptic Digests/Peptides}

\author{
Zoltan Takats, Justin M. Wiseman, Demian R. Ifa and R. Graham Cooks
}

Cold Spring Harb Protoc; doi: 10.1101/pdb.prot4993

\begin{tabular}{|c|c|}
\hline $\begin{array}{r}\text { Email Alerting } \\
\text { Service }\end{array}$ & Receive free email alerts when new articles cite this article - click here. \\
\hline $\begin{array}{l}\text { Subject } \\
\text { Categories }\end{array}$ & $\begin{array}{l}\text { Browse articles on similar topics from Cold Spring Harbor Protocols. } \\
\text { Characterization of Protein Complexes ( } 83 \text { articles) } \\
\text { Characterization of Proteins (208 articles) } \\
\text { Chromatography ( } 47 \text { articles) } \\
\text { Chromatography, general (134 articles) } \\
\text { High-Throughput Analysis, general (155 articles) } \\
\text { High-Throughput Chromatography (4 articles) } \\
\text { Liquid Chromatography ( } 51 \text { articles) } \\
\text { Mass Spectrometry (78 articles) } \\
\text { Protein Identification and Analysis (202 articles) } \\
\text { Proteins and Proteomics, general (575 articles) } \\
\text { Proteome Analysis (56 articles) } \\
\text { Proteomics (66 articles) } \\
\text { Structural Analysis ( } 16 \text { articles) }\end{array}$ \\
\hline
\end{tabular}

